\title{
Contemporary pedagogy within surgical education websites: a review of the literature
}

\author{
Jain B. Pillai ${ }^{1}$, Reg Dennick ${ }^{2}$ \\ ${ }^{1}$ Department of Cardiothoracic Surgery, University Hospital of Coventry and Warwickshire, UK \\ ${ }^{2}$ Medical Education Unit, University of Nottingham, UK
}

Correspondence: Jain B. Pillai, Department of Cardiothoracic Surgery, University Hospital of Coventry and Warwickshire, Clifford Bridge Road, Coventry CV2 2DX, United Kingdom. Email: jain_freeman@hotmail.com

\begin{abstract}
Objectives: To investigate if current surgical web based education provides appropriate learning experiences, viewed through the frameworks of contemporary pedagogy.

Methods: Using defined exclusion and inclusion criteria, we searched the medical literature for papers on surgical web education. We analysed the instructional designs and web utilities to uncover the pedagogical frameworks underpinning the teaching and learning experiences used.

Results: Twenty five eligible studies were analysed and descriptive summary statistics were produced. Traditional teacher-centred behaviourist pedagogy underpinned 52\% $(n=12)$ of the studies. Socio-constructive pedagogy that fosters dialogue, collaboration and co-construction of

present in $9 \%(n=2)$. Examples of good web implementation of various pedagogic frameworks are described.

Conclusions: Pedagogic shortcomings and underutilisation of Web 2.0 technology are revealed in current web surgical education. Educators must shift away from the traditional focus on teacher centred content delivery. The web remains impersonal. Social constructivism, collaboration and two way communications need more focus. We propose some recommendations and suggest that these should apply to any medical education website for pedagogic rigour. These highlight the exciting opportunities for future research in assessing educationally balanced web technology as a solution for consistent 'out of operating room' training in surgery amidst increasing service commitments.
\end{abstract} knowledge was seen in $21 \%(n=5)$. A humanistic context was seen in $21 \%(n=5)$. Learner generated content was
Keywords: Web learning, Internet learning, pedagogy, surgical education, medical education

\section{Introduction}

Our intention in this paper is to investigate how current surgical web based education utilises established pedagogical principles.

We believe that the web medium must become an integral element in the delivery of surgical education in the future. This necessity arises from major working pattern changes that doctors have recently faced which restrict the time available for teaching and learning. Legal limitation of doctors' working hours in Europe and the USA, the consequent conflict between training and service delivery and the constant search for efficient use of resources have become powerful interlinked forces that demand new ways of teaching and learning. The deep rooted surgical tradition of learning by apprenticeship and lectures, embodying the
Halsteadian concept of a hundred years ago, has not evolved. ${ }^{1}$ Teaching by traditional methods may well prove less productive for achieving consistently high learning experiences in the current environment. In a Scottish basic surgical trainee survey on the 40 hour European working week, $81 \%$ of trainees felt that only $40 \%$ or less of their time was actually spent in surgical training compared to service provision. $^{2}$ In the 80 hour working week of the American surgical residency programs, $40 \%$ of residents felt that more than half of their time was spent on pure service delivery. ${ }^{3}$ In this new environment, there is less trainee-trainer contact time for education.

In this context, we feel that the web medium offers a compelling solution. Invented by British scientist Tim 
Berners Lee in 1989, the web has now become a social force and has changed forever the way people on Earth communicate. We believe that the internet holds the promise to reintegrate the trainer and the trainee across physical and temporal boundaries, and provides a teaching and learning 'solution' at either's convenience. But the Internet has not integrated effectively with serious education, in comparison to its widespread use in commercial and social spheres of human activity.

“Today's generation of students have been termed millennial learners or the Net Generation, because they have been raised in a media-rich environment and live in an information-centric world. Many of these students have surfed the Internet since early adolescence, have purchased clothing and concert tickets on the Internet and communicate with peers via multiple instant messaging windows....". ${ }^{4}$

The UK-based committee of inquiry into the changing learner experience cautions: "The world they (young people) encounter in higher education has been constructed on a wholly different set of norms. Characterised broadly, it is hierarchical, substantially introvert, guarded, careful, precise and measured. The two worlds are currently coexisting, with present-day students effectively occupying a position on the cusp of change. They aren't demanding different approaches; rather they are making such adaptations as are necessary for the time it takes to gain their qualifications. Effectively they are managing a disjuncture, and the situation is feeding the natural inertia of any established system. It is, however, unlikely to be sustainable in the long term. The next generation is unlikely to be so accommodating and some rapprochement will be necessary if higher education is to continue to provide a learning experience that is recognised as stimulating, challenging and relevant". ${ }^{5}$

This combination of changes in surgical working times and the revolutionary influences of the web is making web based surgical education increasingly attractive. The introduction of the European Working Time Directive (EWTD) is an opportunity to modernise services. It provides an opportunity to emphasise the need for strong clinical leadership and involvement in medical education. ${ }^{6} \mathrm{We}$ believe that traditional onsite surgical training must be critically re-examined to separate the 'cognitive phase' from the 'performance phase'. To increase the efficiency and value of the limited training time in hospital we believe 'training' in the first phase must be conducted by innovative means such as the web.

However, we realise that effective web education must be based on the principles of contemporary pedagogy. This forms the basis of our investigation, which is to evaluate web based surgical education from a pedagogical perspective. We are not aware of any previous reviews on this aspect of surgical web education. Contemporary pedagogy has evolved over the last few decades towards a learner centred approach including 'active learning. ${ }^{7}$ No longer can learners be considered passive recipients of knowledge and skills, but instead should be seen as active participants in the learning process. Learning is such a complex human endeavour that it defies any single unifying theory. Nevertheless, we will use a range of learning theories to provide us with a vocabulary and a conceptual framework for interpreting the examples of learning that we observe on surgical web sites. ${ }^{8}$

We will be primarily focussing on the ability of web education to deliver Miller's 'knows' and 'knows-how' stages.' This covers theoretical foundations, patient assessment, decision making and prioritising, procedural steps with pitfalls and complications, including their management. We are excluding the hands-on learning of the 'shows' and 'does' stages because that involves an additional and entirely distinct set of technology beyond the scope of pure web platforms. Moreover only a firm grounding in the first two stages will allow junior to mid-level surgical trainees to acquire a strong specialist knowledge base to make the transition into the advanced levels of surgery with confidence.

To sum up the above threads, therefore, our research aim is to investigate and understand how web based surgical learning uses contemporary pedagogy. For this, our research objectives are:

1. What are the underlying pedagogical frameworks that can be observed in current examples of surgical web based education?

2. How do the types of these pedagogies compare to those recommended in contemporary educational principles?

3. How are available web technology utilities being used to produce desirable educational outcomes and what examples can be described?

4. What recommendations can be proposed to improve the use of web technology for surgical education?

\section{Methods}

A literature search was used to gather the evidence for this project and hence ethical approval was not required. The literature search was limited to studies published from 2000 onwards (to June 2010). The Internet and web based technology came 'of age' only in the late 1990s. We believe that earlier data sets would not be generalisable to 2010 and for modelling the near future. We used the search engines of Pub Med and ISI Web of Science. We used only those available as full text articles.

The initial raw sample search used the following keywords. For web based sites, the keywords included: elearning, online learning, internet learning, web learning, Web 2.0, computer, information technology, and virtual learning. For surgical and medical education, the keywords included: surgery, surgical, medical education, and health 
education. To broaden the search, we also used "education" alone. For pedagogy, the keywords included: pedagogy, pedagogical, and constructivism.

The following primary exclusion criteria were used to enrich and focus the material for the primary research question: computer simulators (other than 2D-multimedia and animation for simulating realism); 3D environments and virtual reality such as Second Life; synchronous use of Internet technology (the European Working Time Directive with variable off-duty periods make surgical e- learning more suited to an asynchronous approach); issues of accessibility (the web is becoming a way of life globally and this should not be a decisive factor); Learning management systems such as Moodle, Blackboard, FirstClass, WebCT, etc. (These are technical delivery and organisational issues rather than pedagogical issues); reusable learning object (RLO) models, SCORM, etc - (RLOs represent the next stage in standardisation of pedagogical ideals, 'massification', and resource optimisation).

Secondary inclusion and exclusion criteria were used to further refine the search sample and improve applicability and generalisation. The inclusion criteria used are surgical specialities, institutional studies, academic bodies, learning / teaching model \& method investigations, focus on design of online learning environment, focus on learner participation, focus on learning styles, discussion of pedagogy, and in general a fuller educational package focus via the web. The exclusion criteria used are non-surgical (medical) specialities (unless it has strong web message), very small numbers of learners $<20$, Allied health professionals including Nursing, weak description of web design and utilities with main focus on results, main focus on online assessment/MCQs, or a unilateral focus on a specific web tool such as wiki, pod casts, forum, etc.

A secondary search of 'multimedia' in surgical education was also done, using the additional keyword multimedia. This was undertaken as, we feel, multimedia is pivotal to understanding surgical anatomy for surgical procedures. Guided by Mayer and Moreno ${ }^{10}$ who defined the theory of cognitive overload in multimedia learning, a theoretical framework that best explains the full pedagogic potential of any multimedia material, we felt that the web's increasing ability to deliver rich multimedia deserved further focussed analysis.

\section{Web pedagogy mapping and analysis}

The description in each paper was then critically analysed, against a set of contemporary pedagogical frameworks, to evaluate the web design and uncover the educational approaches that currently support web based surgical education. 'Pedagogic data' was thus generated by mapping the pedagogy found in the identified web-sites to the following learning theories: traditional didactic (behaviourist), cognitive constructivist, social constructivist, situated, humanistic. This data was tabulated under 'manifested web pedagogy' within Table 1 . The data thus acquired was then analysed and summary statistics produced.

The following description of distinct pedagogical perspectives gave us the objectivity to critically analyse the educational environment(s) in each of the papers.

\section{Behaviourist perspective}

Here, observable behaviour rather than internal thought process is the locus of learning. The environment, not the individual learner, shapes behaviour. Behaviourist instructional strategies rely on the development of a set of instructional sequences (content) with predetermined outcomes, and have been the primary approach to teaching since the mid- sixties. Seels and Glasgow described the process as "defining what is to be learned, designing and refining (and reinforcing) the instruction until the objectives (assessment) are met". ${ }^{11}$ This behaviourist stimulus-response orientation has dominated much of the traditional teacher-centred Instructional design. Pedagogically, this propagates a 'passive' and arguably superficial learning approach.

\section{Cognitive constructivist perspective}

Cognitive constructivism is based on learning from internal cognitive processes rather than by absorption of information from the external world (behaviourism). Here the learner is the most active component of the learning process and builds their knowledge and understand on the basis of previously acquired material. Teachers facilitate awakening of understanding in the learner, and do not simply transmit it. This requires varied active learning strategies and facilitation of authentic and interactive activities of exploration, questioning, experimentation, examining mistakes, use of autonomy and creative problem solving, etc. The eventual meaning is constructed actively by the individual learner, and he/she then makes sense of the experience. It focuses on concept development, deep understanding, and creativity.

\section{Social constructivist perspective}

This is rooted in Vygotsky's emphasis on the importance of social interaction and dialogue for the development of higher cognitive functions. ${ }^{12}$ Here knowledge is coconstructed when individuals engage socially in talk and activity about a shared task. Learning occurs through engaging, incorporating, and critically exploring the views of others, and new possibilities of interpretation are opened through the interaction. ${ }^{13}$ Here learning is a cooperative as well as a collaborative social dialectic process.

\section{Situated learning perspective}

One cannot separate the learning process from the situation in which learning is presented. Barab and Duffy suggest a socio-psychological view where every effort is made to make the learning activity as authentic to the social context in which the knowledge or skill is normally embedded. ${ }^{14}$ Such 
situations include case based learning; problem based learning and cognitive apprenticeships. ${ }^{15}$

\section{Humanistic theory}

This is based on the unlimited human potential for growth and the desire to achieve 'self-actualisation'. Abraham Maslow focuses on human motivation as a hierarchy of needs that include belonging, self-esteem, and selfactualization. ${ }^{16}$ Carl Rogers' characteristics of personcentred learning include personal involvement, selfinitiative, self -valuation and meaningful engagement. ${ }^{17}$

\section{Andragogy}

Malcolm Knowles introduced the term: the art and science of adult learning. ${ }^{18}$ Adult learners are self-directing and capable of determining their own needs. Andragogy is firmly rooted in Humanistic Theory. It may tend to 'minimize' or rather refocus the dominating role of the teacher.

\section{Web pedagogy exemplars}

We will highlight the web methods that successfully illustrate how to provide these learning experiences. These serve to provide meaningful practical insight to the various pedagogical perspectives.

\section{Web-learning tools analysis}

Each paper was also analysed in terms of the following weblearning tools made available in its design; static text/images, rich multimedia, video/animation/audio, asynchronous discussion boards, Web 2.0, wiki, blogs, podcast, 'just in time' links, links to resources, mobile learning, cognitive/learning style options, topic search/content mapping, and non MCQ assessment/Script concordance. These qualitative details are also tabulated in Table 1.

\section{Results}

Initial raw search using the keywords 'web learning', 'internet learning' and 'online learning' yielded about 1805 results. This was narrowed to 664 studies with the keywords 'medical education' or 'health education'. When further focused to 'surgery' and 'pedagogy', it yielded about 70 results. Applying our inclusion and exclusion criteria, our search protocol resulted in 25 final studies that qualified for inclusion in our research project. These studies are detailed in Table 1. Eight randomised controlled trials are included. ${ }^{19-26}$ Four publications from surgical professional bodies are found. ${ }^{26,27,28,29}$ Table 1 gives the breakdown of sample size in individual papers within our study population. Sample size information was present in 17 studies, to give a total of 1474 predominantly surgical professionals.
One meta-analysis and one systematic review ${ }^{30,31}$ were the only two significant studies of this type to be found in medical education (Table 1). These studies included all medical and allied health professionals. The focus was mainly on concrete outcomes using the knowledge gain measures from test results of web based medical education. The predominant tool was the multiple choice question. Cook found that though web based instruction is undoubtedly effective for knowledge learning, it is neither superior nor inferior to traditional teaching methods. ${ }^{31}$ The review by Chumley-Jones ${ }^{30}$ also demonstrated no significant differences in knowledge gains by web based interventions.

Using the defined pedagogic frameworks, the 'pedagogic data' breakdown is shown within columns 5 to 9 of Table 1 . Analysis of pedagogic data revealed the following summary statistics that are displayed in Table 2. It is evident that overlapping pedagogical styles in the delivery of learning are apparent in web-sites. Fifty two percent of studies were clearly dominated by the traditional didactic approach where content delivery was the focus. The design of 'packaged lecture/content', followed by MCQ was the most common. Forty three percent of the studies met the cognitive constructivist pedagogy criteria in which participants build up their understanding by interacting with authentic case-based clinical situations and are able to work through the clinical cycle receiving suggestions and feedback as they progress.

Twenty one per cent of the studies had a social constructivist flavour, in which there were opportunities for online discussion either with peers or instructors or both. Elements of Maslow's humanistic perspective ${ }^{16}$ were seen in these studies ${ }^{3,4,7,11,13}$ comprising $21 \%$ of the group. Efforts at self-directed learning were evident in $13 \%$ while learner generated content was seen in $9 \%$.

The data for specific web tools employed in creating the learning environment are shown in Table 1. This data analysis yielded the results that are as shown in Table 2 . The use of wikis, blogs and podcasts were found to be nonexistent despite the fact that most of these tools have been available since 2004 . There is a $17 \%$ prevalence of discussion board use. Certainly text and static image based web technology, echoing its humble origins, still persists in as much as $48 \%$ of web screens. The more bandwidth demanding rich multimedia is present in about $52 \%$ of web learning sites.

In our analysis, cognitive and learning styles options were seen in $22 \%$ of sites. Topic search and index maps were available in $13 \%$ of the study population. This reflects the prevalence of 'adaptive design' within a humanistic or learner-centred pedagogy. 


\begin{tabular}{|c|c|c|c|c|c|c|c|c|}
\hline \multirow{2}{*}{$\begin{array}{l}\text { Author, } \\
\text { year, } \\
\text { country }\end{array}$} & \multirow{2}{*}{$\begin{array}{l}\text { Participant } \\
\text { speciality }\end{array}$} & \multirow{2}{*}{$\begin{array}{l}\text { Study type, size, } \\
\text { duration }\end{array}$} & \multirow{2}{*}{ Approach, web tools and materials } & \multicolumn{4}{|c|}{ Manifested web pedagogy } & \multirow{2}{*}{$\begin{array}{l}\text { Learner } \\
\text { adaptive } \\
\text { design }\end{array}$} \\
\hline & & & & Behaviourist & $\begin{array}{l}\text { Cognitive } \\
\text { constructivism }\end{array}$ & $\begin{array}{l}\text { Social } \\
\text { constructivism }\end{array}$ & Others & \\
\hline $\begin{array}{l}\text { Peska } \\
2010 \\
\text { USA }^{40}\end{array}$ & $\begin{array}{l}\text { Osteopathic } \\
\text { medicine, } \\
\text { gen surgery, } \\
\text { orthopaedics }\end{array}$ & $\begin{array}{l}\text { RCT } \\
63 \\
8 \text { weeks }\end{array}$ & $\begin{array}{l}\text { Discussion board with patient care scenarios. } \\
\text { Text based assignments and group collaboration and } \\
\text { summation }\end{array}$ & No & N/A & $\begin{array}{l}\text { Yes } \\
\text { Discussion board }\end{array}$ & $\begin{array}{l}\text { Authentic, } \\
\text { contextual, } \\
\text { adult learning }\end{array}$ & No \\
\hline $\begin{array}{l}\text { Fordis } \\
2010 \\
\text { USA }^{19}\end{array}$ & $\begin{array}{l}\text { Primary care } \\
\text { physicians, CME } \\
\text { on } \\
\text { heart disease }\end{array}$ & $\begin{array}{l}\text { RCT } \\
97 \\
2 \text { weeks }\end{array}$ & $\begin{array}{l}\text { Web Video CME (Multiple sessions) versus single } \\
\text { lecture. } \\
\text { Email for feedback, multimedia combination options } \\
\text { (video, audio, text, slides) }\end{array}$ & No & Minimal & $\begin{array}{l}\text { Yes / limited } \\
\text { Optional } 45 \mathrm{~min} \text { web } \\
\text { conference with polling } \\
\text { slides (synchronous) }\end{array}$ & $\begin{array}{l}\text { Spaced vs bolus } \\
\text { learning (cognitive } \\
\text { instructional theory) }\end{array}$ & $\begin{array}{l}\text { Yes } \\
\text { Multimedia } \\
\text { options, } \\
\text { reference } \\
\text { links }\end{array}$ \\
\hline $\begin{array}{l}\text { Pugh } \\
2009 \\
\text { USA }^{27}\end{array}$ & $\begin{array}{l}\text { Surgical educa- } \\
\text { tors } \\
\text { committee on } \\
\text { education }\end{array}$ & $\begin{array}{l}\text { Review } \\
\text { US surgical } \\
\text { residency }\end{array}$ & $\begin{array}{l}\text { ANGEL learning management system. curriculum } \\
\text { with textbook and journal } \\
\text { links, videos, link to other websites, quiz, } \\
\text { discussion board }\end{array}$ & $\begin{array}{l}\text { Yes } \\
\text { All pre- } \\
\text { prescribed } \\
\text { content }\end{array}$ & No & $\begin{array}{l}\text { Yes - Discussion board } \\
\text { post at least once during } \\
\text { rotation - moderated } \\
\text { discussion of issues that } \\
\text { arise during ward care }\end{array}$ & $\begin{array}{l}\text { Contextual and } \\
\text { humanistic (with ward } \\
\text { issues) }\end{array}$ & No \\
\hline $\begin{array}{l}\text { Larvin } \\
2009 \\
\text { UK }^{28}\end{array}$ & $\begin{array}{l}\text { UK Surgical } \\
\text { e-Education } \\
\text { Royal Colleges of } \\
\text { Surgeons (RCS) }\end{array}$ & Review & $\begin{array}{l}\text { e-STEP : RCS England } \\
\text { BeST : RCS Glasgow } \\
\text { BST Online : RCS Edinburgh } \\
\text { case based studies, multimedia (AV, animation), } \\
\text { digital mobile media, topic search, } \\
\text { image bank, MCQs }\end{array}$ & No & Yes & $\begin{array}{l}\text { Yes } \\
\text { Discussion board with } \\
\text { Salmon e-moderation, } \\
\text { anonymity of posts. }\end{array}$ & $\begin{array}{l}\text { Humanistic, } \\
\text { pastoral support, } \\
\text { learner generated } \\
\text { content }\end{array}$ & $\begin{array}{l}\text { Yes } \\
\text { Search } \\
\text { facility in } \\
\text { e-STEP }\end{array}$ \\
\hline $\begin{array}{l}\text { Wu } \\
2009 \\
\text { USA }^{44}\end{array}$ & $\begin{array}{l}\text { Senior residents } \\
\text { gen surgery }\end{array}$ & $\begin{array}{l}\text { Prospective } \\
7\end{array}$ & $\begin{array}{l}\text { Practise based, self-assessment, accuracy of initial } \\
\text { clinical impression and final worked up diagnosis. } \\
\text { All consults logged, text only. Accuracy scores } \\
\text { calculated }\end{array}$ & No & Yes & No & $\begin{array}{l}\text { Reflective practice, } \\
\text { self-directed learning, } \\
\text { learner generated }\end{array}$ & N/A \\
\hline $\begin{array}{l}\text { Kerfoot } \\
2008 \\
\text { USA }^{20}\end{array}$ & $\begin{array}{l}\text { Surgery - } \\
\text { urology }\end{array}$ & $\begin{array}{l}\text { RCT } \\
211 \\
10 \text { weeks }\end{array}$ & $\begin{array}{l}\text { Bolus versus Spaced web education. Text based case } \\
\text { scenarios. Spaced education initiated by emails, with a } \\
\text { link to web material. } \\
\text { MCQ, feedback answers. }\end{array}$ & Yes & Minimal & No & $\begin{array}{l}\text { Cognitive processes \& } \\
\text { memory retention }\end{array}$ & No \\
\hline
\end{tabular}




\begin{tabular}{|c|c|c|c|c|c|c|c|c|}
\hline \multirow{2}{*}{$\begin{array}{l}\text { Author, } \\
\text { year, } \\
\text { country }\end{array}$} & \multirow{2}{*}{$\begin{array}{l}\text { Participant } \\
\text { speciality }\end{array}$} & \multirow{2}{*}{$\begin{array}{l}\text { Study type, size, } \\
\text { duration }\end{array}$} & \multirow{2}{*}{ Approach, web tools \& materials } & \multicolumn{4}{|c|}{ Manifested web pedagogy } & \multirow{2}{*}{$\begin{array}{l}\text { Learner } \\
\text { adaptive } \\
\text { design }\end{array}$} \\
\hline & & & & Behaviourist & $\begin{array}{l}\text { Cognitive } \\
\text { constructivism }\end{array}$ & $\begin{array}{l}\text { Social } \\
\text { constructivism }\end{array}$ & Others & \\
\hline $\begin{array}{l}\text { Risucci } \\
2008 \\
\text { USA }^{29}\end{array}$ & $\begin{array}{l}\text { Surgical residents } \\
\text { Yr 1, via surgical } \\
\text { program } \\
\text { directors }\end{array}$ & $\begin{array}{l}\text { Observational. } \\
\text { 31of } 250 \text { available } \\
\end{array}$ & $\begin{array}{l}\text { Web curriculum. Case based interactive scenarios. } \\
\text { Toolbar access to charts, reports. Embedded just in } \\
\text { time refs. } \\
\text { History taking, 'perform' physical exam,' order' tests, } \\
\text { 'recommend' treatment. } \\
\text { All text based feedback available for actions and } \\
\text { choices made. }\end{array}$ & No & Yes & No & $\begin{array}{l}\text { Adult learning } \\
\text { (motivation- just in } \\
\text { time ref links). } \\
\text { Humanistic: ability to } \\
\text { 'restart'/'tensions' as } \\
\text { case progressed. }\end{array}$ & No \\
\hline $\begin{array}{l}\text { Chenkin } \\
2008 \\
\text { Canada }^{21}\end{array}$ & $\begin{array}{l}\text { Emergency } \\
\text { medicine } \\
\text { residents and } \\
\text { staff physicians }\end{array}$ & $\begin{array}{l}\text { RCT } \\
\text { Non inferiority } \\
21\end{array}$ & $\begin{array}{l}\text { Procedural skill teaching. } \\
\text { Web module versus didactic teaching. } \\
\text { (Ultrasound guided vascular access - central line, } \\
\text { arterial line and peripheral iv). } \\
\text { Video, animation, text, MCQ }\end{array}$ & Yes & No & No & None & None \\
\hline $\begin{array}{l}\text { Corrigan } \\
2008 \\
\text { Ireland }^{37}\end{array}$ & $\begin{array}{l}\text { Medicine } \\
\text { final year }\end{array}$ & $\begin{array}{l}\text { Trial } \\
\text { Non } \\
\text { randomised } \\
115 \\
1 \text { year }\end{array}$ & $\begin{array}{l}\text { SURGENT - problem based clinical scenarios with } \\
\text { realism in approach. Long and short cases. Access to } \\
\text { labs, radiology. Clinical photos. Interpret and } \\
\text { manage. Interactive - results vary per actions. } \\
\text { Feedback }\end{array}$ & No & Yes & No & Situated & None \\
\hline $\begin{array}{l}\text { Bevea } \\
2008 \\
\text { Canada }^{33}\end{array}$ & $\begin{array}{l}\text { Family practice } \\
\text { residents on ENT } \\
\text { skill }\end{array}$ & $\begin{array}{l}3 \text { group non RCT } \\
25 \\
15 \text { min web } \\
\text { module }\end{array}$ & $\begin{array}{l}\text { Skill learning - Particle repositioning manoeuvre in } \\
\text { vertigo. Web module vs classroom lecture vs small } \\
\text { group clinical instruction. } \\
\text { Slides, images, text. No audio-video. quiz }\end{array}$ & Yes & No & $\begin{array}{l}\text { None } \\
\text { in web module }\end{array}$ & None & No \\
\hline $\begin{array}{l}\text { Maddaus } \\
2008 \\
\text { USA }^{42}\end{array}$ & $\begin{array}{l}\text { Surgery residents } \\
\text { (Thoracic, } \\
\text { bariatrics, } \\
\text { oncology, } \\
\text { pediatrics, critical } \\
\text { care) }\end{array}$ & $\begin{array}{l}\text { Observational } \\
2 \text { years }\end{array}$ & $\begin{array}{l}\text { WebCT / Vista: Re-integrating and de-constructing } \\
\text { clinical rotation competencies into web course } \\
\text { modules. Multimedia (not clearly specified), mainly } \\
\text { text based, Online lectures PDF, cases, resource links, } \\
\text { assignments - Pre - Post MCQ / Oral exam }\end{array}$ & Yes & $\begin{array}{l}\text { Yes } \\
\text { Partial }\end{array}$ & $\begin{array}{l}\text { No online discussion } \\
\text { board. } \\
\text { But instead uses collabo- } \\
\text { ration via hybrid face to } \\
\text { face discussions oppor- } \\
\text { tunistically during ward / } \\
\text { theatre encounters. }\end{array}$ & $\begin{array}{l}\text { Humanistic: } \\
\text { Residents feedback } \\
\text { used to alter the design } \\
\text { - learners as co- } \\
\text { developers and } \\
\text { reviewers }\end{array}$ & No \\
\hline $\begin{array}{l}\text { Smolle } \\
2007 \\
\text { Austria }^{39}\end{array}$ & $\begin{array}{l}\text { Medical students. } \\
\text { Thoracic trauma }\end{array}$ & $\begin{array}{l}\text { Observational } \\
41\end{array}$ & $\begin{array}{l}\text { Interactive content, diagrammatic animated simula- } \\
\text { tion for enhanced case based scenarios. } \\
4 \text { modes of presentation - physiology, injuries details, } \\
\text { treatment effects, random injuries for management }\end{array}$ & No & $\begin{array}{l}\text { Yes } \\
\text { System responds to } \\
\text { treatments selected }\end{array}$ & No & $\begin{array}{l}\text { None } \\
\text { Cases can go wrong or } \\
\text { right }\end{array}$ & $\begin{array}{l}\text { Yes } \\
4 \text { content } \\
\text { presentation } \\
\text { styles }\end{array}$ \\
\hline
\end{tabular}




\begin{tabular}{|c|c|c|c|c|c|c|c|c|}
\hline \multirow{2}{*}{$\begin{array}{l}\text { Author, } \\
\text { year, } \\
\text { country }\end{array}$} & \multirow{2}{*}{$\begin{array}{l}\text { Participant } \\
\text { speciality }\end{array}$} & \multirow{2}{*}{$\begin{array}{l}\text { Study type, size, } \\
\text { duration }\end{array}$} & \multirow{2}{*}{ Approach, web tools \& materials } & \multicolumn{4}{|c|}{ Manifested web pedagogy } & \multirow{2}{*}{$\begin{array}{l}\text { Learner } \\
\text { adaptive } \\
\text { design }\end{array}$} \\
\hline & & & & Behaviourist & $\begin{array}{l}\text { Cognitive } \\
\text { constructivism }\end{array}$ & $\begin{array}{l}\text { Social } \\
\text { constructivism }\end{array}$ & Others & \\
\hline $\begin{array}{l}\text { Dance } \\
2007 \\
\text { USA }^{43}\end{array}$ & $\begin{array}{l}\text { Medical students, } \\
\text { Surgery rotation. } \\
28\end{array}$ & Observational & $\begin{array}{l}\text { Web cased case discussion. Alternative to fear of } \\
\text { 'evaluation' on ward rounds. } \\
3 \text { groups, } 9 \text { students each with } 2 \text { facilitators } \\
\text { 2weeks }\end{array}$ & No & None & $\begin{array}{l}\text { Yes } \\
\text { Case discussion, } \\
\text { interlaced with inpatient } \\
\text { issues }\end{array}$ & $\begin{array}{l}\text { Humanistic } \\
\text { less apprehension }\end{array}$ & No \\
\hline $\begin{array}{l}\text { Kallet } \\
2007 \\
\text { USA }^{22}\end{array}$ & Surgery & $\begin{array}{l}\text { Randomised } \\
93 \\
8 \text { weeks }\end{array}$ & $\begin{array}{l}\text { WISE-MD. } \\
\text { Realistic video \& animations for learning on a } \\
\text { 'surgical bloodless field'. Intra-operative video clips, } \\
\text { resource links, text, script concordance testing. }\end{array}$ & No & $\begin{array}{l}\text { Yes } \\
\text { Script concordance } \\
\text { testing }\end{array}$ & No & $\begin{array}{l}\text { ? Web equivalent to } \\
\text { Cognitive Apprentice- } \\
\text { ship }\end{array}$ & No \\
\hline $\begin{array}{l}\text { Kerfoot } \\
2006 \\
\text { USA }^{20}\end{array}$ & $\begin{array}{l}\text { Medical Students } \\
\text { in } \\
\text { Urology, } 4 \\
\text { medical schools. }\end{array}$ & $\begin{array}{l}\text { RCT } \\
286 \\
2 \text { weeks }\end{array}$ & $\begin{array}{l}\text { Web vs clinical teaching } \\
\text { Text based clinical scenarios. } \\
\text { MCQ on management with } \\
\text { feedback }\end{array}$ & Yes & No & No & None & None \\
\hline $\begin{array}{l}\text { Friedl } \\
2006 \\
\text { Germany }^{24}\end{array}$ & $\begin{array}{l}\text { Medical students } \\
\text { and junior } \\
\text { residents. } \\
\text { Cardiac surgery }\end{array}$ & $\begin{array}{l}\text { RCT } \\
126\end{array}$ & $\begin{array}{l}\text { La Medica. } \\
\text { Rich web multimedia vs printed text/image. Complex } \\
\text { spatial anatomy learning in surgery. } \\
\text { Video, audio, animation, images, } 2 \mathrm{D} / 3 \mathrm{D} \text { model, MCQ }\end{array}$ & $\begin{array}{l}\text { Yes } \\
\text { partially } \\
\text { structured } \\
\text { instruction }\end{array}$ & $\begin{array}{l}\text { Yes } \\
\text { Interactive multime- } \\
\text { dia }\end{array}$ & No & None & $\begin{array}{l}\text { Situated } \\
\text { cognition }\end{array}$ \\
\hline $\begin{array}{l}\text { Friedl } \\
2006 \\
\text { Germany }^{25}\end{array}$ & $\begin{array}{l}\text { Junior residents. } \\
\text { Cardiac surgery }\end{array}$ & $\begin{array}{l}\text { RCT } \\
3 \text { groups } \\
195\end{array}$ & $\begin{array}{l}\text { La Medica. } \\
\text { Differing multimedia instructional methods \& } \\
\text { constant amount of content. Interactive with animate } \\
\text { or mark on Images vs structured multimedia vs } \\
\text { text/image in print }\end{array}$ & $\begin{array}{l}\text { Yes } \\
\text { In structured } \\
\text { multimedia }\end{array}$ & $\begin{array}{l}\text { Yes } \\
\text { In } \\
\text { interactive } \\
\text { multimedia }\end{array}$ & No & None & $\begin{array}{l}\text { Situated in } \\
\text { interactive } \\
\text { multimedia } \\
\text { (realism) }\end{array}$ \\
\hline $\begin{array}{l}\text { Ferguson } \\
2006 \\
\text { USA }^{34}\end{array}$ & $\begin{array}{l}\text { Surgical residents } \\
\text { Year } 1 \& 2\end{array}$ & $\begin{array}{l}\text { Retrospective } \\
\text { control study } \\
19 \\
1 \text { year }\end{array}$ & $\begin{array}{l}\text { BeST Resident } \\
\text { multimedia, presumed text } \\
\text { (tools not clearly explained) } \\
\text { Influence of web learning on ABSITE surgical board } \\
\text { exams }\end{array}$ & Yes & No & No & None & None \\
\hline $\begin{array}{l}\text { Servais } \\
2006 \\
\text { USA }^{38}\end{array}$ & $\begin{array}{l}\text { Medical students } \\
\text { surgery clerkship }\end{array}$ & Observational & $\begin{array}{l}\text { Case based realistic adaptive testing. } 25-35 \text { web pages } \\
\text { per case scenario. Multimedia, text, images, radiology, } \\
\text { labs. Topic index available, resource links. MCQs } \\
\text { presented as per responses. Text feedback prompts } \\
\text { and links. }\end{array}$ & No & Yes & No & $\begin{array}{l}\text { Situated } \\
\text { Realism. } \\
\text { Humanistic } \\
\text { (Non-threatening } \\
\text { environment for } \\
\text { complex issues) }\end{array}$ & $\begin{array}{l}\text { Yes } \\
\text { Topic index } \\
\text { for depth of } \\
\text { learning }\end{array}$ \\
\hline
\end{tabular}




\begin{tabular}{|c|c|c|c|c|c|c|c|c|}
\hline \multirow{2}{*}{$\begin{array}{l}\text { Author, } \\
\text { year, } \\
\text { country }\end{array}$} & \multirow{2}{*}{$\begin{array}{l}\text { Participant } \\
\text { speciality }\end{array}$} & \multirow{2}{*}{$\begin{array}{l}\text { Study type, size, } \\
\text { duration }\end{array}$} & \multirow{2}{*}{ Approach, web tools \& materials } & \multicolumn{4}{|c|}{ Manifested web pedagogy } & \multirow{2}{*}{$\begin{array}{l}\text { Learner } \\
\text { adaptive } \\
\text { design }\end{array}$} \\
\hline & & & & Behaviourist & $\begin{array}{l}\text { Cognitive } \\
\text { constructivism }\end{array}$ & $\begin{array}{l}\text { Social } \\
\text { constructivism }\end{array}$ & Others & \\
\hline $\begin{array}{l}\text { Gold } \\
2005 \\
\text { USA }^{26}\end{array}$ & $\begin{array}{l}\text { Thoracic surgery } \\
\text { directors } \\
\text { association } \\
\text { curriculum } \\
\text { project. } \\
\text { Residents }\end{array}$ & $\begin{array}{l}\text { RCT } \\
138 \\
3 \text { years }\end{array}$ & $\begin{array}{l}\text { CD-ROM (videos)/ web hybrid. } \\
\text { Content presentation \& selection options for depth } \\
\text { or breadth. } 75 \text { learning modules in } 13 \text { textbook like } \\
\text { sections. AV narration, radiology, ref links. } \\
\text { or as } 12 \text { clinical scenarios }\end{array}$ & No & Yes & No & $\begin{array}{l}\text { Situated } \\
\text { (Case scenarios with } \\
\text { videos) }\end{array}$ & $\begin{array}{l}\text { Yes } \\
\text { Cognitive } \\
\text { and Learn- } \\
\text { ing styles } \\
\text { catered }\end{array}$ \\
\hline $\begin{array}{l}\text { Healy } \\
2005 \\
\text { Ireland }^{35}\end{array}$ & $\begin{array}{l}\text { Medical students, } \\
\text { Surgery rotation }\end{array}$ & $\begin{array}{l}\text { Observational } \\
148 \\
14 \text { week per } \\
\text { group, } 2 \text { years }\end{array}$ & $\begin{array}{l}\text { iCAL-SURG. } \\
\text { Lectures notes on web as PowerPoint, word, pdf. } \\
\text { Questions and answers. } \\
\text { Pictorial OSCE and questions. Correct answers for } \\
\text { assignments posted a day after }\end{array}$ & Yes & No & No & None & No \\
\hline $\begin{array}{l}\text { Meier } \\
2005 \\
\text { USA }^{36}\end{array}$ & $\begin{array}{l}\text { Year 1 } \\
\text { residents } \\
\text { general surgery }\end{array}$ & $\begin{array}{l}\text { Observational } \\
16 \\
4 \text { months }\end{array}$ & $\begin{array}{l}\text { Learning common postop complications (MI, Sepsis, } \\
\text { Pulmonary edema). } \\
\text { Web content to complement simulator learning. ?Text } \\
\text { based (web tools poorly described) }\end{array}$ & Yes & No & No & None & No \\
\hline $\begin{array}{l}\text { Bernado } \\
2004 \\
\text { Brazil }^{32}\end{array}$ & $\begin{array}{l}\text { Medical students } \\
\text { surgery }\end{array}$ & $\begin{array}{l}\text { Observational } \\
56 \\
5 \text { weeks }\end{array}$ & $\begin{array}{l}\text { CD web hybrid. } \\
\text { Videos, text reading, images. } \\
\text { Questions and quiz. } \\
\text { Feedback answers }\end{array}$ & Yes & No & $\begin{array}{l}\text { Yes - rudimentary } \\
\text { Messages are posted on a } \\
\text { discussion blackboard. } \\
\text { Reply from tutor posted } \\
\text { on FAQ. }\end{array}$ & None & No \\
\hline $\begin{array}{l}\text { Cook } \\
2008 \\
\text { USA }^{31}\end{array}$ & $\begin{array}{l}\text { Multi } \\
\text { institutional } \\
\text { Multi specialities } \\
\text { in medicine and } \\
\text { surgery in USA }\end{array}$ & $\begin{array}{l}\text { Systematic review } \\
\text { \& meta-analysis. } \\
201 \text { eligible } \\
\text { studies from } 1990 \\
\text { to } 2008 \text { era }\end{array}$ & $\begin{array}{l}\text { Web arm vs no web (traditional). } \\
\text { Various web utilities. } \\
\text { Focus on knowledge gain effectiveness via web } \\
\text { education. Satisfaction \& behaviour modification also } \\
\text { looked at. }\end{array}$ & Variable & Variable & Variable & Variable & Variable \\
\hline $\begin{array}{l}\text { Jones } \\
2002 \\
\text { USA }^{30}\end{array}$ & $\begin{array}{l}\text { Medical and } \\
\text { surgical. } \\
\text { Multi } \\
\text { institutional }\end{array}$ & $\begin{array}{l}\text { Review and } \\
\text { meta-analysis } \\
76 \text { eligible studies } \\
\text { from } 1996 \\
\text { to } 2002 \text { era }\end{array}$ & $\begin{array}{l}\text { Web utilities not individually described } \\
\text { knowledge gain assessed }\end{array}$ & N/A & N/A & $\mathrm{N} / \mathrm{A}$ & N/A & N/A \\
\hline
\end{tabular}




\section{Pedagogical mapping}

We will not qualitatively elaborate further on those studies and web-sites that we have identified as using a traditional didactic pedagogy of text, static images and assessment other than to positively acknowledge that this method of learning may be suitable for individuals with particular 'learning styles' ${ }^{32-36} \mathrm{We}$ will qualitatively elaborate next on studies and web-sites that manifest the other more contemporary pedagogies. Table 1 has specific details under manifested web pedagogy and learner adaptive design.

Table 2. Pedagogy in surgical websites

\begin{tabular}{lc}
\hline Pedagogy & $\mathrm{n}(\%)$ \\
\hline Learning theories & \\
Traditional didactic (behaviourist) & $12(52)$ \\
Cognitive constructivism & $10(43)$ \\
Social constructivism & $5(21)$ \\
Humanistic & $5(21)$ \\
Situated & $14(61)$ \\
Self-directed learning & $3(13)$ \\
Learner generated content & $2(9)$ \\
Web tools & \\
Static text / images & $11(48)$ \\
Rich multimedia - video, audio, animation & $12(52)$ \\
Asynchronous discussion board & $4(17)$ \\
Web 2.0, wiki, blogs & 0 \\
Podcasts & 0 \\
'Just in time' links & $3(13)$ \\
Link to resources & $6(26)$ \\
Mobile learning & $1(4)$ \\
Cognitive or learning style options & $5(22)$ \\
Topic search / content mapping & $3(13)$ \\
Non MCQ assessment / script concordance & $1(4)$ \\
\hline & \\
\hline
\end{tabular}

\section{Cognitive constructivist design}

The web learning site by Risucci ${ }^{29}$ provides an exemplar for using an interactive case based approach. The learner's web journey is embedded in a real life situation on the ward where the learner interacts with patient scenarios proceeding through the clinical cycle of history taking, physical exam, ordering tests, reviewing of results and recommending treatment. The scenarios have 'just-in-time' reference links for feedback and guidance, in addition to other static links to learning materials. The progress of the case varies depending on learner inputs. This creates an authentic and situated context in addition to being cognitively supported by timely feedback and suggestions for further reading and reflection. The whole scenario is further enveloped in a Humanistic context in that if the case management is going wrong, the learner has the option to 're-set' and start again, without the fear of summative assessment.

The web design by Corrigan ${ }^{37}$ and Servais ${ }^{38}$ operate on similar lines. The WISE-MD e-learning environment by Kalet $^{22}$ expands on this theme by adding the situational realism of surgery by using rich multimedia. Here video and animation are added to the case scenarios to create a 'bloodless' operative scene. This resulted in an improvement in 'self-efficacy' and led to increased comfort in interacting with the consultant surgeons in real operating rooms in about $38 \%$ of students. The La Medica e-learning site by Friedl ${ }^{24}$ is an exemplar of the kind of research that investigates the advantages of good pedagogy. Here the use of rich interactive multimedia with $3 \mathrm{D}$ animations allows the spatial relationships of surgical anatomy to be better understood, leading to significantly increased procedural task and knowledge competence as a second assistant in theatre, as assessed by the consultant operating surgeon. Here, multimedia was compared to exactly similar content given as print media and pictures.

The design by $S$ molle ${ }^{39}$ provided learning on chest trauma using interactive complex diagrammatic animations. Themed modules (chest and lung physiology, treatment options, injury patterns and treatment effects, random cases) were available to the learner to select from. This caters to different learning styles. Learner satisfaction was $82.9 \%$. $17 \%$ expressed the importance of the trial and error process of getting to understanding how to manage an acute surgical scenario.

\section{Social constructivist pedagogy}

A closer look at social constructivist pedagogy, characterised by co-operation and collaboration, reveals pedagogic nuances. The innovative study by Peska and Lewis ${ }^{40}$ uses a discussion board with 5 groups of 3-5 students each. These surgical students interact and come up with a final written report of a case scenario solution within 8 weeks although the amount of student-student interaction, critical analysis of 'knowledge' and modification of learning achieved were not available from the data published. However, the study by $\mathrm{Pugh}^{27}$ revealed that the surgical program directors felt that the time and effort needed for the discussion board was not a 'time-saver' and was rarely used. On the other hand the e-STEP website of the Royal College of Surgeons of England $^{28}$ was exemplary in that it followed Salmon's 5 stage model of e-moderation. ${ }^{41}$ Their website statistics also showed the common phenomenon of the 'lurkers', those who prefer to read and reflect on others' posts without actively contributing themselves. This complicates social constructivist issues and raises the importance of differing learning styles.

\section{Humanistic pedagogy}

Some studies provide some examples of humanistic pedagogy where individual learner's needs are emphasised. ${ }^{27,28,42 \text {, }}$ ${ }^{43}$ Pugh $^{27}$ describe web environments that allowed surgical residents to post issues that arose during routine ward care for a moderated discussion. This was an extra facility in addition to their site's otherwise didactic aspect. Larvin ${ }^{28}$ refers to the availability of pastoral support on a discussion 
board. Maddaus ${ }^{42}$ describes surgical residents who were involved in expressing their learning needs and actively participating in the design of their own e-learning web site. This approach led to a distinct change in the resident population behaviour with almost all fully utilizing and engaging with the e-learning environment. Non-threatening learning environments of acute surgical scenarios, such as those exemplified by Ricussi ${ }^{29}$ and Smolle ${ }^{39}$ provide the humanistic blanket to learners and allow better assimilation of learning.

\section{Self-directed learning}

The following studies ${ }^{42-44}$ illustrate examples of learner motivation and sites that encourage self-directed learning. Surgical residents in the design by $\mathrm{Wu}^{44}$ used their e-learning site to log data of initial diagnostic impressions and later final proven diagnoses, to reflect, learn and improve clinical practice. In the study of Maddaus, ${ }^{42}$ residents themselves helped align the e-site to resident directed needs. In the study by Dance, ${ }^{43}$ surgical students organized into groups and posted and discussed case scenarios with peers and senior residents on a discussion board. 50\% subsequently found that this was beneficial to their learning experience.

\section{Mobile or m-learning}

The only study that took the leap forward into the way of life of the 'digital natives' was the Royal College of Surgeons of England e-STEP learning site that had information that could be downloaded into mobile digital devices. ${ }^{28}$

\section{Situated}

The frequency of situated, authentic and contextual content in web sites was seen in about $61 \%$ of the study population where video and multimedia of surgical processes was common. For a surgeon, one of the most crucial understandings required is that of surgical anatomy. The La Medica e-learning site by Friedl ${ }^{24}$ demonstrates the powerful nature of multimedia that can be provided on the internet. Multimedia was defined by Clark and Mayer ${ }^{45}$ as a medium containing both text and graphics.

\section{Multimedia}

Rich multimedia was seen in about $52 \%$ of our studies. Our secondary search result on multimedia in medical education with a pedagogic focus is detailed in Table 3 .

Exploring the pedagogic concepts behind multimedia use, Dubrowski ${ }^{46}$ studied how 12 medical students actually made use of surgical videos to learn surgical knot tying. It clearly showed a difference in the perceptions of what teachers felt as the 'important steps' compared to the segments in the video that students felt to be more important and actually viewed for learning. Students preferred slow motion sequences rather than real time speed. The slow motion was able to better 'de-construct' the surgical steps. Dilullo ${ }^{47}$ studying 260 medical students found that videos increased self-efficacy in 59\% in achieving good dissections in anatomy. Similar scenario exists when surgical trainees receive less operating time exposure. $\mathrm{Choi}^{48}$ reviewed the characteristics of 25 surgical anatomy websites sites. They found evidence of cognitive constructivism and interactivity with multimedia in only about $15 \%$ of sites. They concluded that animations, layering, zooming and rotation of graphics facilitated the understanding of spatial anatomy. Supplemental clinical photographs and operative images gave contextual motivation for plain text. Refocussing on our research sample, amongst the 12 studies with multimedia tool provision, only 4 studies ${ }^{22,24,28,39}$ (33\%) demonstrated the nuances described by Choi ${ }^{48}$ The web design by Friedl ${ }^{24}$ is an excellent example of this with the ability to mark on the cardiac surgical image or animation, which corresponds to an intraoperative step. This later increased the self-efficacy in theatre.

Finally, we found that in only 2 papers did we see a combination of pedagogies being synergistically applied. The study by Maddaus ${ }^{42}$ used social constructivism in a hybrid fashion with face to face discussion rather than web based discussion board. The study by Larvin (e-Step) had a good combination of pedagogies supporting each other. ${ }^{28}$ The study by Pugh made early but uncommitted steps with implementation of social constructivism. ${ }^{27}$

\section{Discussion}

This study has highlighted the significant variations in the implementation of web based education within various surgical programs. A mix of pedagogies exists within these e- learning environments with a preponderance of the more traditional didactic methods where content is pre-packaged, consumed and assessed. Major studies ${ }^{30,31}$ to date indicate a pre-occupation with recording web learning outcomes in terms of knowledge gains, and are conspicuous by the absence of any data on their pedagogical basis. Reassured by the web's ability to secure knowledge gains ${ }^{30,31}$, we believe other pedagogic considerations must be emphasised in future. The poor prevalence of social constructivist pedagogy is an important starting point to focus our thoughts. The web medium remains isolated from the 'human touch'. Two way communications, with the visual and audio cues of people concerned are lacking. Heavy emphasis remains on impersonal content and knowledge transmission (though an important element) as judged from the high behaviourist incidence. We believe a multi pedagogic perspective and sequence should play a pivotal part in giving websites a chance to become an enduring teaching and learning medium for trainees. Web education should focus on the processes of learning instead of just content delivery. 
Table 3. Summary of critical papers on multimedia in medical education

\begin{tabular}{|c|c|c|c|c|c|}
\hline $\begin{array}{l}\text { Author, } \\
\text { Year, } \\
\text { Country }\end{array}$ & $\begin{array}{l}\text { Participant } \\
\text { speciality }\end{array}$ & $\begin{array}{l}\text { Study type, } \\
\text { size, } \\
\text { duration }\end{array}$ & Reasons for study/background & Outcome assessment & Manifested pedagogy \\
\hline $\begin{array}{l}\text { Choi } \\
2008 \\
\text { New } \\
\text { Zealand }\end{array}$ & $\begin{array}{l}\text { Anatomy. } \\
\text { for surgical } \\
\text { learning }\end{array}$ & $\begin{array}{l}\text { Review. } \\
25 \text { eligible } \\
\text { websites } \\
\text { evaluated }\end{array}$ & $\begin{array}{l}\text { Critical review of e-anatomy resources for } \\
\text { effectiveness for surgical anatomy } \\
\text { learning. } \\
\text { Inclusion: entire body anatomy sites only. } \\
\text { Exclusion: specialist or organ based } \\
\text { anatomy sites. } \\
\text { To define good website features for } \\
\text { learning. }\end{array}$ & $\begin{array}{l}\text { Only } 5 \text { websites (out of possible 34) } \\
\text { were classed as most useful for } \\
\text { learning. } \\
\text { Desirable Features : } \\
\text { The ability of computer graphics to } \\
\text { use layering, zoom, and rotation of } \\
\text { 3d models to facilitate spatial } \\
\text { anatomy understanding. Accom- } \\
\text { panying succinct notes. Podcast } \\
\text { tutorials and audio-visual lectures. } \\
\text { Good road map / hierarchical } \\
\text { indexing of contents }\end{array}$ & $\begin{array}{l}\text { Cognitive constructivism } \\
\text { Realism/situated cognition/ } \\
\text { contextual. } \\
\text { Adult learning. Self-efficacy. } \\
\text { Reflection. } \\
\text { Cognitive styles - field } \\
\text { dependant and field inde- } \\
\text { pendent (navigation) } \\
\text { Learning styles - not all are } \\
\text { visual learners (audio provi- } \\
\text { sion). }\end{array}$ \\
\hline $\begin{array}{l}\text { Dilullo } \\
2006 \\
\text { USA }^{47}\end{array}$ & $\begin{array}{l}\text { Medical } \\
\text { students }\end{array}$ & 260 & $\begin{array}{l}\text { Ability to perform successful anatomy } \\
\text { dissections, without damaging structures. } \\
\text { Multimedia effectiveness over lack of time } \\
\text { for personal tutor instruction. } \\
\text { Video clips - short }<120 \text { sec, } 2-7 \text { sequences } \\
\text { per dissection module. } \\
\text { Text only narrative/no voice over }\end{array}$ & $\begin{array}{l}75 \% \text { improved self-efficacy for } \\
\text { dissections } \\
58 \% \text { felt text helped clarify video } \\
\text { Majority wanted audio narration } \\
\text { instead of text }\end{array}$ & $\begin{array}{l}\text { Pavio's dual coding effect } \\
\text { Instructional theory }\end{array}$ \\
\hline $\begin{array}{l}\text { Dubrowski } \\
2005 \\
\text { Canada }^{46}\end{array}$ & Surgery & & $\begin{array}{l}\text { How to effectively use video instruction } \\
\text { for surgical instruction and learning } \\
\text { procedural skills. Instead of 'practising' on } \\
\text { live patients in operating theatre. } \\
\text { Videos in } 2 \text { modes - slow motion \& } \\
\text { normal speed. Segmented the } 240 \text { sec clips } \\
\text { into } 28 \text { bins @10sec each. To look at } \\
\text { which parts of skill the trainees focussed } \\
\text { (and what trainers need to know) }\end{array}$ & $\begin{array}{l}\text { Slow motion preferred over real } \\
\text { time speed. } \\
\text { Trainee and trainer focus did not } \\
\text { agree or match. } \\
\text { (Trainee viewed more 'looping of } \\
\text { sutures' whereas Trainers felt } \\
\text { 'suture placement' and 'hand } \\
\text { movements' were more important) }\end{array}$ & Instructional theory \\
\hline
\end{tabular}

We will summarise our arguments on this very process of presentation and creation of content. Behaviourist 'static content' predominated in $52 \%$ of sites. Between various surgical websites, similar topics need similar content. If each institution were to create its own basic content, we believe this 'content re-creation' (especially text and simple image based) becomes a wasteful effort. It is also resource heavy. Importantly, web based e-learning environments should not become a 'Fast-Food' service for content. In an era of instant information access on the web, learners have become more impatient and have a tendency to 'surf and 're- surf the net for readymade information. We believe that educators should not continue to maintain an undue focus on content creation driven by a content search engine mentality. Surgical web sites should be more than just online surgical text books.

Content can be more effectively presented by 'just-intime' hyperlinks to time honoured textbooks (or access to electronic subscription of a prescribed textbook), all within a local curriculum. This would be more resource efficient. Just in time links were present in only $13 \%$ in our study. Alternatively, content can be accessed via index/topic search /content maps. This facility was only present in $13 \%$. More novel types of content should be individually produced.
Podcasts, found to be underutilised in this study, are a powerful method for 'creative' and 'humanising' content presentation and imparting a realistic feel to the online learning environment. The power of the human voice has been beautifully described by Cambell. ${ }^{49}$

“There's also considerable value in what I call the 'explaining voice', the voice that performs understanding. The explaining voice doesn't just convey information; it shapes, out of a shared atmosphere, an intimate drama of cognitive action in time. The explaining voice conveys micro-cues of hesitation, pacing, and inflection that demonstrate both cognition and metacognition. When we hear someone read with understanding, we participate in that understanding, almost as if the voice is enacting our own comprehension. In other words, the explaining voice trains the ear to listen not just for meaning but for evidence of the thought that generates meaning. Photographs are undeniably powerful, and perhaps a picture is worth a thousand words, but a few words uttered by a dear voice may be worth the most of all".

Moving on to the visual cues, rich multimedia was present in just half of our study population while Interactive rich multimedia was present even less, at a third of the multimedia. The latter increases the self-efficacy of the trainees as shown by Kalet $^{22}$ and Friedl ${ }^{24}$. Edited multimedia 
of surgical scenarios and procedures should also take precedence. The multimedia study by Dubrowski ${ }^{46}$ gives an educator valuable insight into the needs of the surgical learner. The learner watching a skill executed at 'real speed' by the expert (at his Bloom's 'automatic' level on the psychomotor domain), ${ }^{50}$ may not be as easily learnable and transferable, as the experts might imagine. The study essentially gives valuable insight into critical planning while designing multimedia, rather than 'shoot and upload' raw image approaches. The exemplars of cognitive constructivist educational methods (present in less than half of study population) described in the results section bring out the more creative use of readily available resources. Welldesigned multimedia content can significantly support the 'shows how' phase of skill acquisition. The web holds great potential for better constructive learning, moving beyond the conventional 'see one, do one' or 'assist and watch' approaches of traditional surgery. We believe the conventional is both time consuming, educationally unsound, and also fails to deal with the problem that 'the eye does not see what the mind does not know'.

Next, the arrival of Web 2.0 and social collaborative tools have not left a significant mark on surgical education, judging by the $25 \%$ prevalence in our study. Discussion Boards were found in $17 \%$. An equivalent to a Web 2.0 instrument, its prevalence may be related with the fact that it has existed for more than 2 decades in its alternative mail based internet platform. Another exciting tool that is nonexistent in our data is the use of wikis. Wikis uphold the Vygotskian concept of socially constructed knowledge creation. Blogs are another 'discussion media' that are easier to use. Again it was not in vogue in our surgical study sample. The Salmon model - Table 4 shows the Salmon's 5 stage model which provides the much needed and perhaps unappreciated insight and objectivity to analyse online social constructivist pedagogy, when presented on the web. ${ }^{41} \mathrm{We}$ feel therefore this needs a brief elaboration here for the benefit of readers.

Stages 1 to 3 achieve 'knowledge cooperation' while stages 4 to 5 achieve 'knowledge collaboration'. The latter stages complete the full social constructivist pedagogy, which would de-construct to the following: "thinking and responding to other's ideas, widening own viewpoints and appreciating differing perspectives, offering ideas, having them criticised or expanded on, reshaping or abandoning them, in the light of peer discussion" - Rowntree. ${ }^{51}$ This higher pedagogy needs dedicated threading and development of the posts with e-moderation. This level of detail was not present in the study by Peska and Lewis. ${ }^{40}$ Also the 'free ride seeker' or 'lurker' must be identified and encouraged to participate. This raises a salient research issue of how to effectively achieve the intended pedagogy from collaborative web tools, and reminds that the mere provision of a web tool does not translate to pedagogy. The study by $\mathrm{Pugh}^{27}$ is a case in point. "It is ironic that what some take to be dehumanizing technology (web) may actually need teachers to be more empathetic and considerate". 52

In general, why is the use of 'Web 2.0', which is in vogue as a social connective technology, not being reflected in medical educational use of the web? Sandars and Schroter ${ }^{53}$ and Sandars and Morrison ${ }^{54}$ give contemporary evidence from a horizontal survey of medical professionals' use of Web 2.0 tools. More than $50 \%$ of the e-educators were not accustomed to these newer web tools educationally, though more than $50 \%$ of Net Generation medical students appreciated their use in learning. This discordance between 'digital immigrants' and 'digital natives' respectively leaves us to conclude that 'education about education' is more relevant now than ever before. The role of informed eeducators is pivotal. Educators need to understand the dynamics of online social constructivism. Dedicated time is needed for these web affordances. When appropriately harnessed, we believe that it leads to more learner satisfaction, understanding and creates a community of participation that is seeded online and which grows 'on-ground' in the hospital and in theatre.

\section{Recommendations}

What we have found therefore is a non-uniform, often isolated or non-connected means of employing different pedagogic strategies on the web. The exemplars in our results section do illuminate the many good web elaborations of specific and different pedagogies. But many of them lack the provision of 'conversation or dialogue' as would happen in a traditional classroom. Acknowledging and drawing on Beetham and Sharpe, ${ }^{55}$ we propose that a combination of different and a logical sequence of contemporary pedagogies should be used in surgical web based environments to create the best possible educationally sound learning experiences. Otherwise, we believe this can disenchant the learners from the full potential of achieving learning via the medium of the web.

\section{Using pedagogy}

1. Learners should engage in social constructivist dialogue, co-construct and create new understanding and bring to the surface contradictions and ambiguities. Authentic daily clinical (ward and intensive care), operative, emergency and on call problems in the learner's (and/or trainer's) own experience can form the substrate of these dialogues via emoderated discussion boards and blogs. In addition, nonclinical but allied training issues can also be addressed through Web 2.0 tools, providing a supportive humanistic and non-threatening environment. Learner or trainer generated regular podcasts are another means to present the above context in an engaging manner. This will raise the sense of personal involvement, relevance and collaborative learning. This is what andragogy ${ }^{18}$ is based on. 
Table 4. Salmon's 5 stage model - objectivity for online social constructivist pedagogy

\begin{tabular}{|c|c|c|c|}
\hline Design & $\begin{array}{l}\text { Learning process with } \\
\text { 'staged aims' }\end{array}$ & $\begin{array}{l}\text { e- Moderating } \\
\text { vital aspects }\end{array}$ & $\begin{array}{l}\text { Unique considerations of } \\
\text { 'online talk' }\end{array}$ \\
\hline $\begin{array}{l}\text { 1. Access and motivation } \\
\text { Setting up system and accessing the } \\
\text { web and web tool } \\
\text { Welcoming and Encouraging }\end{array}$ & $\begin{array}{l}\text { Overcoming tech- } \\
\text { nical/computer/ setting up and } \\
\text { connection issues }\end{array}$ & $\begin{array}{l}\text { Provide timely assistance to avoid } \\
\text { frustration and de-motivation }\end{array}$ & \\
\hline $\begin{array}{l}\text { 2. Online socialisation } \\
\text { Sending and receiving messages } \\
\text { Familiarising and providing } \\
\text { 'bridges' between cultural, social } \\
\text { and learning environments } \\
\text { Provision of a 'Bar' or 'Cafe area' in } \\
\text { Learning environment }\end{array}$ & $\begin{array}{l}\text { Creating trust } \\
\text { virtual third culture }\end{array}$ & $\begin{array}{l}\text { Accept 'Lurkers' at this stage } \\
\text { Send 'private emails' to strong dominant } \\
\text { opinionators to encourage democratic } \\
\text { participation of the group. } \\
\text { 'Emotional literacy' vs 'informational } \\
\text { literacy' }\end{array}$ & $\begin{array}{l}\text { Can ask questions without waiting for } \\
\text { turn. Messages are 'neutral' and dis- } \\
\text { linked from Appearance (? young or } \\
\text { old), Race or other visual clues. } \\
\text { Encourages everyone to be 'them- } \\
\text { selves'. Time lag to response allows } \\
\text { more Reflection }\end{array}$ \\
\hline $\begin{array}{l}\text { 3. Information exchange } \\
\text { Searching, personalising software. } \\
\text { Facilitating tasks and conversation } \\
\text { Supporting use of learning } \\
\text { materials }\end{array}$ & $\begin{array}{l}\text { Trust/confidence/fear - posting } \\
\text { own ideas and making it } \\
\text { permanently available as text. } \\
\text { Information volume overload - } \\
\text { not reading all messages, some } \\
\text { read but do not respond, others } \\
\text { go offline }\end{array}$ & $\begin{array}{l}\text { 'Messiness' of the Learning environ- } \\
\text { ment. } \\
\text { Group size }<20 \text { ? Ideal. Need to present } \\
\text { and link data with regular summary. } \\
\text { Acknowledge value of contribution, } \\
\text { verification of information provided. } \\
\text { Avoid excessive FAQ - does not inspire } \\
\text { communication. Focus with clear goals. }\end{array}$ & $\begin{array}{l}\text { Permanent Record of 'talk' - issues } \\
\text { Can pick up or misunderstand threads } \\
\text { Authority and control shifts to } \\
\text { learners for active participation } \\
\text { Easy to 'lurk' or 'browse' without } \\
\text { contributing - issues with differing } \\
\text { learning styles. Cognitive styles } \\
\text { important - FD can get overwhelmed. }\end{array}$ \\
\hline $\begin{array}{l}\text { 4. Knowledge construction } \\
\text { Conferencing and facilitation } \\
\text { process }\end{array}$ & $\begin{array}{l}\text { Thinking and responding to } \\
\text { others ideas. Widening own } \\
\text { viewpoints. Appreciating } \\
\text { differing perspectives. } \\
\text { Vygotsky - offering ideas, } \\
\text { having them criticised or } \\
\text { expanded on, reshaping them in } \\
\text { light of peer discussion. }\end{array}$ & $\begin{array}{l}\text { Rise of 'social clarity' and co- } \\
\text { construction of knowledge. } \\
\text { Selecting problem based cases where no } \\
\text { obvious right answer seen or which need } \\
\text { a series of ideas. } \\
\text { E-moderator must ask more, challenge, } \\
\text { compliment, weave discussions together } \\
\text { or provide fresh strand if going off stray. }\end{array}$ & $\begin{array}{l}\text { E-moderator must gradually reduce } \\
\text { dependence of the group on the } \\
\text { moderator. }\end{array}$ \\
\hline $\begin{array}{l}\text { 5. Development } \\
\text { Providing links outside closed } \\
\text { conferences } \\
\text { Supporting and Responding }\end{array}$ & Deep constructive learning & Set up specific tasks to complete & $\begin{array}{l}\text { Stages } 1,2 \text {, and } 3 \text { - a form of } \\
\text { cooperation } \\
\text { Stages } 4 \text { and } 5 \text { - is true collaboration }\end{array}$ \\
\hline
\end{tabular}

2. Having raised the motivation of the learners, the web environment should take them further. Cognitive Constructivist activities can next assist in reaching the higher ground of deep learning using a variety of e-learning activities such as case-based rich multimedia presentations and interactive scenarios, collaborative assignments via wiki, creating podcasts on specific issues, blood or radiological investigations or critical care parameters for diagnosis, computer aided assessments including script concordance testing.

3. A supportive environment must exist to clarify the facts. Learners can navigate through 'just-in-time' hyperlinks to didactic e-learning resources (text, graphics, multimedia, on-line book chapters, PowerPoint presentations, resource banks) index and curriculum mapped against appropriate learning outcomes.

An ideal web learning environment must be seen as a purposeful journey for deep learning. We emphasize that the educational principle echoed by William Butler Yeats' quote from the last century ${ }^{56}$ remains applicable even for technology driven education in the twenty first century. The web journey should be one that 'lights the fire' in the learner, and not just 'fills the pail'.

\section{Using web design}

Hence to achieve the above, a seamless combination of all the following should make the web learning environment come alive with the human factor.

1. Content is not the start of learning. Contemporary pedagogy suggests problems and discussions are the foundations of lifelong learning. Focus on e-content instruction should give way to knowledge construction.

2. A web based e-learning site should not become a content resource. This would only be akin to recreating summaries of textbooks. This propagates the inertia of the learners and promotes behaviourist and superficial learning.

3. 'Content pre-occupation' can be reduced by just-in-time hyperlinks to accepted major textbooks (electronic subscription or hardback) within a curriculum map. This would be a better use of time and money.

4. There is a need to increase collaborative learning tools on e-learning sites (discussion boards, wikis, and blogs). 
5. Technology provision cannot be left alone to achieve high end social constructivist pedagogy. It requires good understanding of the dialogue stages and dynamic effort on both the part of the learner and the educator.

6. Realism and situated learning in surgery can be enhanced with thoughtfully edited rich multimedia. Such content increases self-efficacy and cannot be obtained by standard didactic educational resources.

7. There should be more use of the power of the human voice. Podcasts are a simple yet powerful and cognitively enabling technology. Its prevalence is absent in our study results.

8. A Humanistic pedagogic context should be provided for better learner motivation and engagement.

9. The role of informed e-educators is vital. Educationally trained surgeons (and other doctors) have a significant opportunity here.

\section{The limitations of this study}

The study is accurate only as far as the original search engine results are. There may be publication bias, as not all existing web education sites may have published data or evaluations about their site. We did not personally review the actual web sites as that would need access rights and was not practically feasible. Our inclusion criteria could be challenged by others. Our study size of 25 papers is not large, even though they represent a broad range within the surgical education community. Our categorization of the eligible studies into pedagogic types is a qualitative interpretation and is also only as accurate as the information that is provided in the original article.

\section{Conclusion}

Our data derived from the surgical literature would indicate that a significant portion of current web learning is not yet functioning as a complete pedagogic package. It may be that the surgical community is not an exception in this respect and that this condition may be found in e-medical education practices in general. Therefore we would suggest that our recommendations would apply to any medical specialty as far as educational principles are concerned. At this point, we are not aware of any other study on web pedagogy analysis. We believe the strength of our study is its potential to elevate awareness and create dialogue about the current inadequacies of web teaching and learning. We hope our paper encourages educators to spend more time appreciating how the next generation of trainees is currently educating itself (with ambient and ubiquitous technology) and how educators have the responsibility to learn to incorporate these media into the curricula. We hope the web will be used with scientific rigour and become an essential part of learning and training, rather than as an optional or secondary method. We believe the web can increase the efficiency of the time spent in training, and provide a more uniform and equally rich experience to all trainees, irrespective of the educational inconsistencies in the traditional hospital service-pressured environment.

Our results thus open up the opportunity for more experimentation and research in the field of pedagogically sound medical e-learning. When appropriate pedagogies are integrated with the social and technological revolutions of the digital age, the following questions need answering. Will it lead to a more satisfying and genuinely acceptable e-learning environment? Can it provide the consistent continuity, better value for time and effort and will it emerge as the main training solution not competing with service commitments? What are the demands imposed on finances, resources and training the trainer commitments in the route to a more rounded pedagogic approach? We are also left with further thoughts. What should be the ideal mix of the many pedagogical approaches made available? How much does this mix depend on the different learning styles as well as self-directed learning abilities, motivation and methods of learners?

One of the authors of this paper is a cardiac surgeon who is personally aware of the frustrations with traditional surgical teaching and learning and who finds the evidence from this research inspirational and motivating. Over ten years later, the following extract of a speech given by Bill Gates to faculty and students of the New York Institute of Technology in 2000 is still ever more relevant: "The web will change Generation I's world as much as television transformed our world after World War II. That is why it is so critical to ensure that new teachers understand how to incorporate technology into their instruction and that teachers have the technological training they want and need. We cannot afford to have any teacher locked out of the greatest library on earth-the Internet." 57

Gorman's article's title "From blood and guts to bits and bytes" ${ }^{\prime \prime}$ just as well encapsulates the message of this paper. If everyone shares this conviction developed in this paper, we hope that web based e-learning in surgery will change the face of the time honoured 'face to face' learning of centuries past. Educational theory, web technology and surgical training will then reach a new equilibrium.

\section{Conflict of Interest}

The authors declare that they have no conflict of interest.

\section{References}

1. Gorman PJ, Meier AH, Rawn C, Krummel TM. The future of medical education is no longer blood and guts, it is bits and bytes. Am J Surg. 2000;180:353-356

2. Skipworth RJE, Terrace JD, Fulton LA, Anderson DN. Basic surgical training in the Era of the European working time directive: what are the problems and solutions? Scott Med J. 2008;53:18-21.

3. Reines H, Robinson L, Nitzchke S, Rizzo A. Defining service and education: the first step to developing the correct balance. Surgery. 2007;142:303-10.

4. Maag M. iPod, uPod? An emerging mobile learning tool in nursing education and students' satisfaction. 23rd Annual ASCILITE Conference; Sydney, Australia; 2006. 
5. JISC. Higher education in a Web 2.0 world. 2009 [cited 20 July 2011]; Available from: http://www.jisc.ac.uk/media/documents/publications/ heweb20rptv1.pdf.

6. Temple J. Time for Training. A review of the impact of the European working time directive on the quality of training. 2010 [cited 12 September 2011]; Available from: http://www.mee.nhs.uk/pdf/JCEWTD_Final\%20 report.pdf.

7. Biggs J, Tang C. Teaching for quality learning at university. Berkshire, England: Open University Press; 2007.

8. Hill WF. Learning: a survey of psychological interpretations. Boston, MA: Allyn \& Bacon; 2002.

9. Miller GE. The assessment of clinical skills competence performance. Acad Med. 1990;65:S63-S67.

10. Mayer RE, Moreno R. Nine ways to reduce cognitive load in multimedia learning. Educational Psychologist. 2003;38:43-52.

11. Seels B, Glasgow Z. Exercises in instructional design. Columbus: Merrill; 1990.

12. Vygotsky LS. Mind in society: The development of higher psychological process. Cambridge: Harvard University Press; 1978.

13. Gergen K. Social construction and the educational process. In: Steffe L, Gale J, editors. Constructivism in education. New Jersey: Erlbaum; 1995.

14. Barab S, Duffy T. From practice fields to communities of practice. In: Jonassen D, Land S, editors. Theoretical foundations of learning environments. New Jersey: Erlbaum; 2000.

15. Brandt B, Farmer J, Buckmaster A. Cognitive apprenticeship approach to helping adults learning In: Flannery D, editor. Applying cognitive learning theory to adult learning. San Francisco: Jossey-Bass; 1993.

16. Maslow AH. Motivation and personality. New York: Harper Collins; 1970.

17. Rogers CR. Freedom to learn for the 80s. Columbus: Merrill; 1983.

18. Knowles MS. The modern practice of adult education: from pedagogy to andragogy. New York: Cambridge Books; 1980

19. Fordis M, King JE, Ballantyne CM. Workshops: a randomized controlled trial internet-based CME with live interactive CME. Comparison of the instructional efficacy. JAMA. 2005;294:1043-1051.

20. Kerfoot BP, Baker H, Jackson TL, Hulbert WC, Federman DD, Oates $\mathrm{RD}$, et al. A multi- institutional randomized controlled trial of adjuvant web-based teaching to medical students. Acad Med. 2006;81:224-230.

21. Chenkin J, Lee S, Huynh T, Bandiera G. Procedures can be learned on the Web: a randomized study of ultrasound-guided vascular access training. Acad Emerg Med. 2008;15:949-54.

22. Kalet AL, Coady SH, Hopkins MA, Hochberg MS, and Riles TS. Preliminary evaluation of the web initiative for surgical education (WISEMD). Am J Surg. 2007;194:89-93.

23. Kerfoot BP. Interactive spaced education versus web based modules for teaching urology to medical students: a randomized controlled trial. J Urol. 2008;179:2356-7.

24. Friedl R, Höppler H, Ecard K, Scholz W, Hannekum A, Ochsner W et al. Multimedia- driven teaching significantly improves students' performance when compared with a print medium. Ann Thorac Surg. 2006;81:1760-6.

25. Friedl R, Höppler H, Ecard K, Scholz W, Hannekum A, Oechsner W, et al. Comparative evaluation of multimedia driven, interactive, and casebased teaching in heart surgery. Ann Thorac Surg. 2006;82:1790-5.

26. Gold JP, Begg WB, Fullerton DA, Mathisen DJ, Orringer MB, Verrier ED. Evaluation of web-based learning tools: lessons learned from the thoracic surgery directors association curriculum project three-year experience. Ann Thorac Surg. 2005;80:802-10.

27. Pugh CM, Watson A, Bell RH Jr, Brasel KJ, Jackson GP, Weber SM, et al. Surgical education in the internet era. J Surg Res. 2009;156:177-182.

28. Larvin M. E-learning in surgical education and training. ANZ J Surg. 2009;79:133-137.

29. Risucci DA, Numann PJ, Welling R, Pfingsten MF, Blair PG, Sachdeva AK. Reactions of surgical program directors to a web-based interactive educational program focusing on cognitive skills. J Surg Educ. 2008;65:470475

30. Chumley-Jones HS, Dobbie A, Alford CL. Web-based learning: sound educational method or hype? A review of the evaluation literature. Acad Med. 2002;77:S86-S93.
31. Cook DA, Levinson AJ, Garside S, Dupras DM, Erwin PJ, Montori VM. Internet-based learning in the health professions - A meta-analysis. JAMA. 2008;300:1181-1196.

32. Bernardo V, Ramos, MP, Plapler H, De Figueiredo LF., Nader HB., Anção, MS, et al. Web-based learning in undergraduate medical education: development and assessment of an online course on experimental surgery. Int J Med Inform. 2004;73:731-42.

33. Beyea JA, Wong E, Bromwich M, Weston WW., Fung K. Evaluation of a particle repositioning maneuver web-based teaching module. Laryngoscope. 2008;118:175-80

34. Ferguson C, Warshaw A. Failure of a web-based educational tool to improve residents' scores on the American board of surgery in-training examination. Arch Surg. 2006;141:414-6.

35. Healy DG, Fleming FJ, Gilhooley D, Felle P, Wood AE, GoreyT, et al Electronic learning can facilitate student performance in undergraduate surgical education: a prospective observational study. BMC Med Educ. 2005;29:5-23.

36. Meier AH, Henry J, Marine R, Murray WB. Implementation of a weband simulation -based curriculum to ease the transition from medical school to surgical internship. Am J Surg. 2005;190;137-140.

37. Corrigan M, Reardon M, Shields C, Redmond H. "SURGENT" - Student e-learning for reality: the application of interactive visual images to problem-based learning in undergraduate surgery. J Surg Educ. 2008;65:120-125.

38. Servais EL, LaMorte WW, Agarwal SS, Moschetti W, Mallipattu SK, Moulton, SL. Teaching surgical decision-making: an interactive, web-based approach. J Surg Res. 2006;134:102-106.

39. Smolle J, Prause G, Smolle-Ju“Ttner FM. Emergency treatment of chest trauma - an e-learning simulation model for undergraduate medical students. Eur J Cardiothorac Surg. 2007;32:644-647.

40. Peska D, Lewis K. Uniform instruction using web-based, asynchronous technology in a geographically distributed clinical clerkship: analysis of osteopathic medical student participation and satisfaction. J Am Osteopath Assoc. 2010;110:135-42

41. Salmon G. E-moderating: the key to teaching and learning online. Oxon: Routledge; 2008.

42. Maddaus MA, Chipman JG, Whitson BA, Groth SS, Schmitz CC Rotation as a course: lessons learned from developing a hybrid online/onground approach to general surgical resident education. J Surg Educ. 2008;65:112-6.

43. Dance M, Jamshidi R. \& O'sullivan P. Facilitated web-based case discussions in surgery. Med Educ. 2007;41:1092-3.

44. Wu B, Dietz P, Bordley JT, Borgstrom D. A novel, web-based application for assessing and enhancing practice-based learning in surgery residency. J Surg Educ. 2009;66:3-7

45. Clark RC, Mayer RE. E-learning and the science of instructions: proven guidelines for consumers and designers of multimedia learning. San Francisco: John Wiley \& Sons; 2008.

46. Dubrowski A and Xeroulis G. Computer-based video instructions for acquisition of technical skills. J Vis Commun Med. 2005;28:150-155.

47. DiLullo C, Coughlin P, D'Angelo M, McGuinness M, Bandle J, Slotkin $\mathrm{EM}$, et al. Anatomy in a new curriculum: facilitating the learning of gross anatomy using web access streaming dissection videos. J Vis Commun Med. 2006;29:99-108

48. Choi ARA, Tamblyn R, Stringer MD. Electronic resources for surgical anatomy. ANZ J Surg. 2008;78:1082-1091.

49. Campbell G. Podcasting in Education. Educause Review. 2005;40:33-46. 50. Bloom BS, Engelhart MD, Furst EJ, Hill WH, Krathwohl DR. Taxonomy of educational objectives. New York: Longmans; 1956.

51. Rowntree D. Teaching and learning online: a correspondence education for the 21st-century.BJET.1995;26:205-215.

52. Knight PT. Being a Teacher in higher education. Buckingham: Open University Press; 2002

53. Sandars J, Schroter S. Web 2.0 technologies for undergraduate and postgraduate medical education: an online survey. Postgrad Med J. 2007;83:759-762.

54. Sandars J, Morrison C. What is the net generation? The challenge for future medical education. Med Teach. 2007;29:85-88. 
Pillai \& Dennick Contemporary pedagogy within surgical education websites

55. Beetham H, Sharpe R. Rethinking pedagogy for a digital age: designing and delivering e-learning. Oxon: Routledge; 2009.

56. del Sol. Constructivist philosophy in education. 2004 [cited 2 May 2010]; Available from: http://delsolschool.org/Constructivist\%20Philosophy
\%20plus\%20LIST.pdf.

57. Leonard B. After generations X and Y comes generation I - Internet generation2000. [cited August 12th 2010]. Available from: http:// findarticles.com/p/articles/mi_m3495/is_1_45/ai_59283651/. 